324

\section{BLS Ambulance Personnel Performance Evaluation}

Peris A, * Ametoli F**

* Department Anaesthesia and Intensive Care, USL 10D

** Medical Training Program

Emergency Medical Services, USL 10H

Florence, Italy

Objective: To demonstrate that non-medical personnel in medical emergency medical systems are more efficient in the application of basic life support (BLS) procedures than are personnel operating in non-medical emergency facilities.

Method: Fifty-six members of non-medical services (Group I) and 48 members of medical services (Group II) were tested on immobilization maneuvers, splinting, and BLS (simulation with and without a practice mannequin).

Results: The Group II operators generally proved to be more able in BLS procedures, immobilization, and splinting.

Conclusion: Because of constant medical supervision, medical services guarantee maintenance of performance levels. In nonmedical facilities, a decline in quality may be prevented only through the use of periodic refresher courses.

\section{5}

\section{Use of Low Earth Orbit Satellite}

\section{Communication Service in the Reconstructive or Subacute Phase of Disaster Relief}

Clements $C L$, *Janson $A M^{* *}$

* Center for Health Communication

Harvard School of Public Health

Boston, Massachusetts, USA

** Department of Histology and Neurobiology

Karolinska Institutet

Stockholm, Sweden

Objective: To demonstrate the suitability of an electronic mail service called HealthNet for use in the reconstructive phase of disasters.

Methods: HealthNet, a microsatellite, links medical centers in the developing world every 12 hours. Its earth stations, personal computers linked to radios, can be briefcase-sized and solar-powered. Its design parameters: affordability, sustainability, and appropriate technology for a variety of different settings, are similar to the long-term communication needs following disasters where real-time communication is not critical.

Results: HealthNet is providing reliable, economical communication service in Africa. Because transmission and reception are via radio, no costs are associated with day-to-day use.

Conclusion: After the acute phase of international disasters such as the Armenian earthquake, donated equipment and services were withdrawn leaving long-term relief and reconstruction efforts without easy access to affordable communication or sources of information. HealthNet is a communication/information service ideally suited for situations such as Somalia.
326

The Role of Professional Judgment in the Assessment of Incoming Calls to EMS Systems

Farand L, * Leprohon J, ** Champagne F, * Contandriopoulos AP, *

Preker $A,{ }^{+}$Kalina $M^{* * *}$

* Health Administration Department,

Universite de Montreal

** Centre for Medical Education, McGill University

**** Urgence Sante

Montreal, Quebec, Canada

+ World Bank

Washington, D.C., USA

Objective: To identify the role and professional judgment, if any, in the assessment of incoming calls to EMS systems.

Methods: Until recently, the assessment of incoming calls to the Montreal EMS system was based entirely on the professional judgment of nurses and physicians. Conceptually, telephone assessment involves three sub-tasks: (1) "triage," or deciding whether or not to send EMS vehicle(s); (2) "prioritization," or deciding about the optimal delay of intervention; and (3) choice of the level of intervention. Performance can be measured in terms of decision-time, sensitivity, and specificity.

A random sample of 1,000 calls was collected. Decisions made by professionals, decision-times, and constraints pertaining to communication (e.g., speech problems) or the actual decision (e.g. refusal) were obtained for each call from tape recordings and dispatching data. Ideal decisions were obtained by consensus of experts, based on emergency department diagnoses and follow-up calls to patients about the evolution of their clinical status during the 48 hours following the call to EMS.

Results: Triage sensitivity was nearly perfect and specificity was $55 \%$. Prioritization sensitivity was $91 \%$ and specificity was $73 \%$. Sensitivity for the choice of intervention level was $83 \%$ and specificity was $82 \%$. Thus, the sensitivity/specificity ratio increased with the "costs" of potential errors. Decision-times varied inversely to levels of urgency and were minimal for vital emergencies. Specificity decreased with shorter decision-times. Constraints on communication corresponded to increased sensitivity, and direct constraints on the decision reduced performance at all levels.

Conclusion: These patterns of performance suggest that professional judgment in this context involves a flexible adaptation of the decision-making process to the characteristics of clinical situations and to the availability of resources while maintaining a high level of safety for the population. 